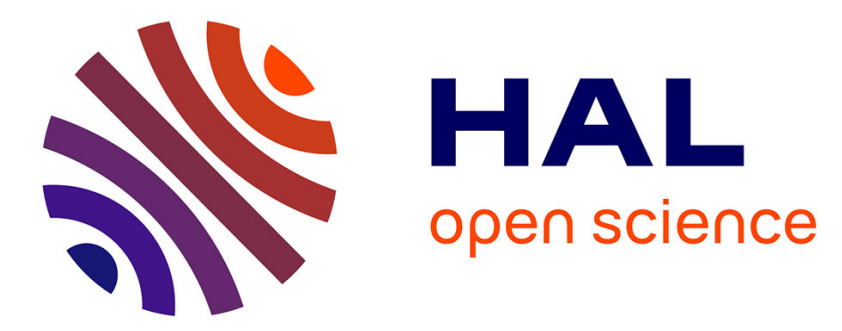

\title{
Stabilization and Control of Delayed Recycling High Order Systems with one Unstable Pole at the Forward Path
}

Juan Francisco Marquez-Rubio, Rocio Jasmin Vazquez-Guerra, Basilio del Muro-Cúellar, Olivier Sename

\section{To cite this version:}

Juan Francisco Marquez-Rubio, Rocio Jasmin Vazquez-Guerra, Basilio del Muro-Cúellar, Olivier Sename. Stabilization and Control of Delayed Recycling High Order Systems with one Unstable Pole at the Forward Path. Asian Journal of Control, 2016, 18 (5), pp.1-13. 10.1002/asjc.1190 . hal-01245424

\section{HAL Id: hal-01245424 \\ https://hal.science/hal-01245424}

Submitted on 19 Jan 2016

HAL is a multi-disciplinary open access archive for the deposit and dissemination of scientific research documents, whether they are published or not. The documents may come from teaching and research institutions in France or abroad, or from public or private research centers.
L'archive ouverte pluridisciplinaire HAL, est destinée au dépôt et à la diffusion de documents scientifiques de niveau recherche, publiés ou non, émanant des établissements d'enseignement et de recherche français ou étrangers, des laboratoires publics ou privés. 


\title{
STABILIZATION AND CONTROL OF DELAYED RECYCLING HIGH ORDER SYSTEMS WITH ONE UNSTABLE POLE AT THE DIRECT PATH
}

\author{
J.F. Marquez-Rubio, R.J. Vazquez-Guerra, B. del Muro-Cuéllar and O. Sename
}

January 14, 2016

\begin{abstract}
In this work the stabilization and control of delayed recycling systems is addressed. Recycling systems are characterized by possessing to main paths named the direct (feedforward) and the recycling (feedback) paths. Such class of systems reuse the energy and/or the partially processed matter increasing the efficiency of the overall process. It is proposed a control methodology for the stabilization and control of this kind of systems. The particular class of systems addressed here contains one unstable pole, $m$ stable poles, a delay term and possible $p$ left half plane zeros $(L H P)$ in the direct path and a delayed stable subsystem in the recycling path. The strategy is based on an asymptotic observer-predictor to estimate the required internal signals. Necessary and sufficient conditions are stated in order to guarantee the stabilization of the proposed scheme, achieving step tracking and step disturbance rejection.
\end{abstract}

\section{INTRODUCTION}

Recycling systems are commonly found in chemical industry, for instance, in a typical plant formed by reactor/separator process, where reactants are recycled back to the reactor [1]. They reuse the energy and the partially processed matter increasing the efficiency of the overall process. In recycling systems a partial feedback of the process output to the input induces a positive feedback, which can give rise to some undesirable effects. Luyben [3] studied the effects of recycle path on dynamics process and their implications to plant-wide control. Taiwo [4], discussed the robust control for recycling plants and proposed the concept of recycle compensation to recuperate inherent process dynamics, i.e. dynamics without recycle. Scali and Ferrari [5] analyzed the problem under same idea. Similar approaches were extended by Lakshminarayanan and Takada [6], and Kwok et. al [7]. 
On the other hand, stability analysis of systems with time delay (and without recycle) together simple controllers is not an easy task, see for instance [8]. Therefore, when significant transport delay is present in recycling systems the control problem becomes more involved. It is known that when recycle path and time delays occur, exponential terms appear in the direct and the recycling paths. In the state space recycling systems with time delay correspond to systems with delays in the input and the state variables. Model approximations have been proposed to remove the exponential terms from the transfer function denominator of a delayed system, such as the method of moments [9] or Pade approximations [10]. Other techniques, such as Taylor series expansion [11], and the seasonal time-series model [7], have been proposed to obtain an approximate model to represent recycling systems. In [12] an approximated model to represent recycling systems by using discrete-time approach is proposed. In turn, such approximated models can be used for stability analysis or control design [13], [5], [14], [15]. A system with time delay and open-loop unstable poles is notably more difficult to control than a system with only open-loop stable poles. For instance, the classical Smith Predictor cannot be used in the case of delayed unstable processes. Introducing recycle in such system would lead to a more difficult (although interesting) problem. Existing modified schemes to Smith Predictor cannot directly be applied to this kind of systems. This is, because the open-loop recycling system is not a system with only time-delay in the direct path but it is a system with internal delay term, i.e., an open loop infinite dimensional system. In order to tackle this problem, we consider here recycling systems composed of a plant with one unstable pole, $m$ stable poles, a delay term and $p$ possible left half plane zeros $(L H P)$ in the direct path and a delayed stable subsystem of order $n$ in the recycling path. The present work intends to extend the results presented in [16] and [17] where recycling systems restricted to unstable first order plants in the direct path are considered. A first attempt to extend these results was presented in [18], dealing with high order plant in the direct path with one unstable pole but without including zeros and without achieving step tracking or step disturbance rejection.

In this work, an observer-predictor based control scheme is proposed in order to stabilize and control recycling systems with delay term in both paths, a stable system in the recycling path and a high order system with one unstable and $m$ stables poles in the direct path. The proposed scheme allows achieving the step tracking and step disturbance rejection. Then, a modification to the scheme is proposed in order to deal with a more general family of plants including $p L H P$ zeros in the direct path. Necessary and sufficient conditions are given for the existence of the proposed stabilizing scheme. It is important to note that, the problem of the stabilization and control of delayed unstable plants even without recycle path is not completely solved. For instance, recent works [21],[22] deal with the stabilization and control of delayed systems with only one unstable pole.

The outline of the paper is as follows. In Section 2 the problem is formulated and the class of systems considered in this work is precised. The general idea of the solutions is also outlined in this section, namely the need of an 
observer-predictor. Section 3 some preliminary stability results are presented. The Section 4 presents the main results. This section is divided in three parts. Firstly in the Subsection 4.1 an observer-predictor scheme to estimate some internal signals of the system is proposed. Then Subsection 4.2 presents the proposed control approach and in the Subsection 4.3, stabilization of recycling systems by considering a high order with an unstable pole and $L H P$ zeros in the direct path is provided. Some simulation results are described in Section 5. Such results illustrate the performance of the proposed control strategy. Finally Section 6 presents some conclusions.

\section{PROBLEM FORMULATION}

Consider the class of recycling system shown in Fig. 1, which can be described as,

$$
Y(s)=\left[\begin{array}{ll}
G_{d}(s) & G_{d}(s) G_{r}(s)
\end{array}\right]\left[\begin{array}{c}
U(s) \\
Y(s)
\end{array}\right]
$$

with,

$$
\begin{aligned}
& G_{d}(s)=G_{1}(s) e^{-\tau_{1} s}=\frac{\alpha}{(s-a)\left(s+b_{1}\right) \ldots\left(s+b_{m}\right)} e^{-\tau_{1} s}, \\
& G_{r}(s)=G_{2}(s) e^{-\tau_{2} s}=\frac{N(s)}{D(s)} e^{-\tau_{2} s}
\end{aligned}
$$

where $G_{d}(s)$, and $G_{r}(s)$ are transfer functions of the direct and the recycling paths, respectively; $\tau_{1}, \tau_{2} \geq 0$ are the time delays associated to $G_{d}(s)$, and $G_{r}(s) . \quad a, b_{i} \in \mathbb{R}^{+}$with $i=1,2, \ldots, m$ i.e., $G_{d}(s)$ is unstable. $N(s)$ and $D(s)$ are polynomials on the complex variable $s$, with the roots of $D(s)$ in $L H P$, i.e., $G_{r}(s)$ is stable. $U(s)$ is the process input and $Y(s)$ is the process output.

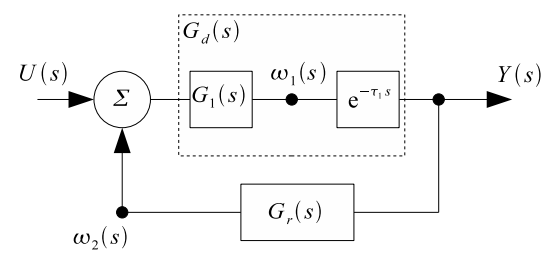

Figure 1: A process with recycle

The transfer function of the recycling system (1) is given by,

$$
G_{t}(s)=\frac{Y(s)}{U(s)}=\frac{D(s) \alpha e^{-\tau_{1} s}}{H_{a u x}(s) D(s)-\alpha N(s) e^{-\left(\tau_{1}+\tau_{2}\right) s}} .
$$

where $H_{a u x}(s)=(s-a)\left(s+b_{1}\right) \ldots\left(s+b_{m}\right)$. Note that exponential terms appear explicitly in the numerator and the denominator of $G_{t}(s)$. Stability of $(3)$ is determined by the roots of its characteristic quasi-polynomial,

$$
Q(s)=H_{\text {aux }}(s) D(s)-\alpha N(s) e^{-\left(\tau_{1}+\tau_{2}\right) s} .
$$


Note that the transcendental term in $Q(s)$ induces an infinite number of roots. Then, for this kind of plants it is not an easy task to conclude about the dynamical behaviour (stability for instance) even in the uncontrolled plant case. Obviously, the related transfer function when the system is controlled with an output feedback becomes more complicated involving more than one transcendental term.

Let us to describe some ideas behind the proposed methodology. With reference to Fig. 1, if the signal $\omega_{2}(s)$ is measured, then we could set,

$$
U(s)=R_{1}(s)-\omega_{2}(s),
$$

obtaining the system shown in Fig. 2. Then it would be possible to design $R_{1}(s)$ as $R_{1}(s)=J(s)\left(R(s)-\omega_{1}(s)\right)$ as in Fig. 3, where $R(s)$ is the input reference. Since $\omega_{1}(s)$ and $\omega_{2}(s)$ are unmeasured internal system signals, an observerpredictor scheme to estimate these variables is developed in the Section 4 . In the following Section some preliminary stability results are presented.

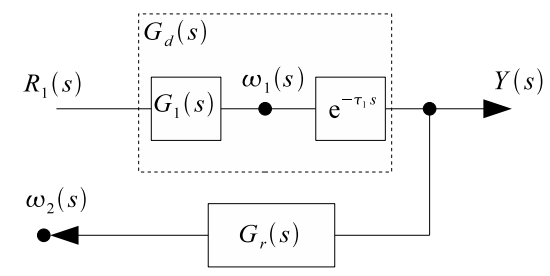

Figure 2: System of Fig. 1 after applying $\mathrm{U}(\mathrm{s})$ in (5)

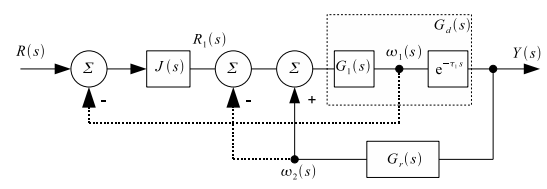

Figure 3: An ideal control structure for the system of Fig. 2

\section{Preliminary stability results}

Consider the system,

$$
G(s)=\frac{Y(s)}{U(s)}=G_{d f} e^{-\tau s}
$$

where

$$
G_{d f}=\frac{\gamma}{(s-a)\left(s+b_{1}\right)\left(s+b_{2}\right) \ldots\left(s+b_{m}\right)}
$$

$a, b_{i} \in \mathbb{R}$, with $a, b_{i}>0 \forall i=1,2, \ldots, m ; \frac{1}{a}-\sum_{i=1}^{m} \frac{1}{b_{i}}>0$ and the static control law,

$$
U(s)=[R(s)-Y(s)] k,
$$


with $R(s)$ as the new input reference.

The following result states the stability condition when a proportional controller is used for unstable delayed systems including one unstable pole, $m$ stable poles and a time delay.

Lemma 1 [20] [21] There exists a gain $k$ such that the closed loop system,

$$
\frac{Y(s)}{R(s)}=\frac{k \gamma e^{-\tau s}}{(s-a)\left(s+b_{1}\right)\left(s+b_{2}\right) \ldots\left(s+b_{m}\right)+k \gamma e^{-\tau s}},
$$

is stable if and only if

$$
\tau<\frac{1}{a}-\sum_{i=1}^{m} \frac{1}{b_{i}}
$$

From condition (9) the size of the delay in order to achieve the closed-loop stability is restricted by the position of the unstable pole and the stable poles in the real axis of the plane $s$.

In the following procedure the set of stabilizing gains $k$ related to Lemma 1 is obtained. First the phase crossover frequency $\omega_{c}$ is given by,

$$
-\tau \omega_{c}+\arctan \left(\frac{1}{a} \omega_{c}\right)-\sum_{i=1}^{m} \arctan \left(\frac{1}{b_{i}} \omega_{c}\right)=0,
$$

where $\omega_{c}>0$. Then, the stabilizing gain $k$ is within the range of,

$$
\frac{a}{\gamma} \prod_{i=1}^{m} b_{i}<k<\frac{a}{\gamma} \prod_{i=1}^{m} b_{i} M
$$

with,

$$
M=\sqrt{\left(1+\left(\frac{1}{a}\right)^{2} \omega_{c}^{2}\right) \prod_{i=1}^{m}\left(1+\left(\frac{1}{b_{i}}\right)^{2} \omega_{c}^{2}\right)} .
$$

On the other hand, consider the transfer function given by,

$$
G(s)=\frac{\alpha\left(s+z_{1}\right) \ldots\left(s+z_{p}\right)}{(s-a)\left(s+b_{1}\right) \ldots\left(s+b_{m}\right)} e^{-\tau_{1} s},
$$

with $p \leq m$ and $z_{1}, \ldots, z_{p}>0$, i.e., $p$ left half plane $(L H P)$ zeros in the direct path of the recycling system and a partition of $G(s)$ as follows,

$$
G(s)=G_{a}(s) e^{-\tau s} G_{b}(s)
$$

where,

$$
\begin{aligned}
G_{a}(s) & =\frac{\alpha}{(s-a)\left(s+b_{1}\right) \ldots\left(s+b_{m-p}\right)}, \\
G_{b}(s) & =\frac{\left(s+z_{1}\right) \ldots\left(s+z_{p}\right)}{\left(s+b_{m-p+1}\right) \ldots\left(s+b_{m}\right)},
\end{aligned}
$$

and a state space representation of $G_{b}(s)$ as $\left(A_{b}, B_{b}, C_{b}, D_{b}\right)$. The following result states the stability condition for the output injection shown in Fig. 4. 
Lemma 2 Consider the system given by (12), the partition (13) and the output injection scheme shown in Fig. 4. There exists $L$ and $k$ such that the closedloop system is BIBO (Bounded Input-Bounded Output) stable if and only if $\tau<\frac{1}{a}-\sum_{i=1}^{m-p} \frac{1}{b_{i}}$.

Proof. Consider the partial output injection shown in Fig. 4 by means of the vector $L$. Then, $L$ should be computed such that,

$$
\sigma\left(s I-A_{b}+L C_{b}\right)=\left\{-z_{1},-z_{2}, \ldots,-z_{p}\right\},
$$

where $\sigma($.$) represents the eigenvalues of, and -z_{1},-z_{2}, \ldots,-z_{p}$ are the zeros of $G_{b}(s)$. Notice that the vector $L$ satisfying (15) involves zero-pole cancellations. Thus, the cancellations are valid since they take place in the left half plane $(L H P)$. With this, we see that the output injection shown in Fig. 4 is reduced to a system of the form (6)-(7), where its stability properties are analyzed in Lemma 1. Hence the result follows.

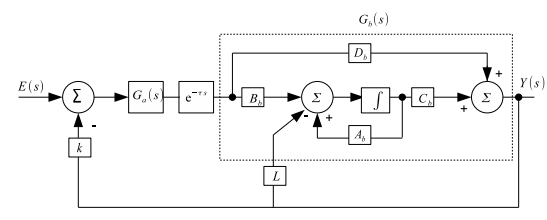

Figure 4: Output injection scheme.

Lemma 3 Consider the delay free model $G_{d f}$ in (6) and a PI/PID controller with an unitary output feedback. Then there exists a stabilizing controller if and only if $0<\frac{1}{a}-\sum_{i=1}^{m-p} \frac{1}{b_{i}}$ for PI, or $0<\sqrt{\frac{1}{a^{2}}+\sum_{i=1}^{m} \frac{1}{b_{i}^{2}}}+\frac{1}{a}-\sum_{i=1}^{m} \frac{1}{b_{i}}$ for PID controller.

Proof. This result is a particular case of the Theorem 1/2, presented in Lee et. al $[21]$, by considering the delay term equal to zero.

\section{MAIN RESULTS}

\subsection{Prediction Strategy}

In order to estimate $\omega_{1}(s)$ and $\omega_{2}(s)$ in Fig. 1 we propose the observer-predictor depicted in Fig. 5. Its convergence is established in the following result.

Theorem 4 Consider the observer-predictor scheme shown in Fig. 5, assuming $G_{r}(s)$ is a stable transfer function and $G_{d}(s)$ defined as in (2a). There exists a constant $k$ such that

$$
\lim _{t \rightarrow \infty}\left[\omega_{j}-\widehat{\omega}_{j}\right]=0
$$




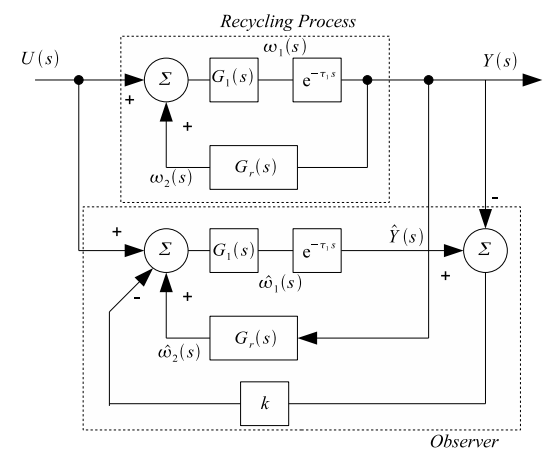

Figure 5: Proposed observer scheme

for $j=1,2$, if and only if

$$
\tau_{1}<\frac{1}{a}-\sum_{i=1}^{m} \frac{1}{b_{i}}
$$

for $i=1,2 \ldots, m$.

Proof. A state space representation of the observer-predictor scheme shown in Fig. 5 is

$$
\begin{aligned}
& \dot{\mathbf{x}}(t)=A \mathbf{x}(t)+A_{1} \mathbf{x}\left(t-\tau_{1}\right)+A_{2} \mathbf{x}\left(t-\tau_{2}\right)+B u(t) \\
& \mathbf{y}(t)=C \mathbf{x}\left(t-\tau_{1}\right)
\end{aligned}
$$

with, $\mathbf{x}(t)=\left[\begin{array}{llll}x_{d}(t) & x_{r}(t) & \widehat{x}_{d}(t) & \widehat{x}_{r}(t)\end{array}\right]^{T}, \mathbf{y}(t)=\left[\begin{array}{ll}y(t) & \widehat{y}(t)\end{array}\right]^{T}, B=$ $\left[\begin{array}{llll}B_{d} & 0 & B_{d} & 0\end{array}\right]^{T}$,

$$
\begin{gathered}
A=\left[\begin{array}{cccc}
A_{d} & 0 & 0 & 0 \\
0 & A_{r} & 0 & 0 \\
0 & 0 & A_{d} & 0 \\
0 & 0 & 0 & A_{r}
\end{array}\right], \\
A_{1}=\left[\begin{array}{cccc}
0 & 0 & 0 & 0 \\
B_{r} C_{d} & 0 & 0 & 0 \\
B_{r} k C_{d} & 0 & -B_{d} k C_{d} & 0 \\
B_{r} C_{d} & 0 & 0 & 0
\end{array}\right], \\
A_{2}=\left[\begin{array}{cccc}
0 & B_{d} C_{r} & 0 & 0 \\
0 & 0 & 0 & 0 \\
0 & 0 & 0 & B_{d} C_{r} \\
0 & 0 & 0 & 0
\end{array}\right], \\
C=\left[\begin{array}{cccc}
C_{d} & 0 & 0 & 0 \\
0 & 0 & C_{d} & 0
\end{array}\right],
\end{gathered}
$$

where $\mathbf{x} \in \mathbb{R}^{p}(p=n+m+1)$ is the state vector, $u \in \mathbb{R}$ is the input, $\mathbf{y} \in \mathbb{R}^{2}$ is the output, $\tau_{1} \geq 0$ and $\tau_{2} \geq 0$ are the time delays in the system. $A_{d} \in$ 
$\mathbb{R}^{(m+1) \times(m+1)}, B_{d} \in \mathbb{R}^{(m+1) \times 1}$, and $C_{d} \in \mathbb{R}^{1 \times(m+1)}$ are matrices and vectors parameters that correspond to the direct path in the process $\left(G_{1}(s)\right)$, given by,

$$
\begin{gathered}
A_{d}=\left[\begin{array}{ccccc}
a & 0 & 0 & \cdots & 0 \\
1 & -b_{1} & 0 & \cdots & 0 \\
0 & 1 & -b_{1} & \cdots & 0 \\
\vdots & \vdots & \vdots & \ddots & \vdots \\
0 & 0 & 0 & \cdots & -b_{m}
\end{array}\right], \\
B_{d}=\left[\begin{array}{c}
\alpha \\
0 \\
0 \\
\vdots \\
0
\end{array}\right], C_{d}=\left[\begin{array}{lllll}
0 & 0 & 0 & \cdots & 1
\end{array}\right]
\end{gathered}
$$

and $A_{r} \in \mathbb{R}^{n \times n}, B_{r} \in \mathbb{R}^{n \times 1}$, and $C_{r} \in \mathbb{R}^{1 \times n}$ are matrices and vectors parameters that corresponds to the recycling path in the process, $\widehat{x}(t)$ is the estimation of $x(t)$.

From Fig. 5 we are strictly interested in the convergence of $e_{\omega_{i}}=\hat{\omega}_{i}-\omega_{i}$ (for $i=1,2)$. However, as $\omega_{1}(s)$ and $\omega_{2}(s)$ are a static functions of the states $x_{d}(t)$ and $x_{r}(t)$, we can equivalently analyze the convergence of $e_{x_{d}}(t)$ and $e_{x_{r}}(t)$ defined in what follows. Defining the state prediction errors $e_{x_{d}}(t)=\widehat{x}_{d}(t)-$ $x_{d}(t), e_{x_{r}}(t)=\widehat{x}_{r}(t)-x_{r}(t)$, and the output estimation $e_{y}(t)=\widehat{y}(t)-y(t)$, it is possible to describe the behavior of the error signals as,

$$
\left[\begin{array}{c}
\dot{e}_{x_{d}}(t) \\
\dot{e}_{x_{r}}(t) \\
e_{y}\left(t+\tau_{1}\right) \\
e_{\omega_{2}}\left(t+\tau_{2}\right)
\end{array}\right]=A_{p}\left[\begin{array}{c}
e_{x_{d}}(t) \\
e_{x_{r}}(t) \\
e_{y}(t) \\
e_{\omega_{2}}(t)
\end{array}\right],
$$

with,

$$
A_{p}=\left[\begin{array}{cccc}
A_{d} & 0 & -B_{d} k & B_{d} \\
0 & A_{r} & 0 & 0 \\
C_{d} & 0 & 0 & 0 \\
0 & C_{r} & 0 & 0
\end{array}\right]
$$

Note that $e_{y}(t)=C_{d} e_{x_{d}}\left(t-\tau_{1}\right)$ and that $e_{\omega_{2}}(t)=C_{r} e_{x_{r}}\left(t-\tau_{2}\right)$. Then, system (19) can be rewritten as,

$$
\begin{aligned}
\dot{e}_{x_{d}}(t)= & A_{d} e_{x_{d}}(t)-B_{d} k C_{d} e_{x_{d}}\left(t-\tau_{1}\right) \\
& +B_{d} C_{r} e_{x_{r}}\left(t-\tau_{2}\right), \\
\dot{e}_{x_{r}}(t)= & A_{r} e_{x_{r}}(t) .
\end{aligned}
$$

As $A_{r}$ is a Hurwitz matrix, the stability of system (20) can be analyzed by considering the reduced dynamics

$$
\dot{e}_{x_{d}}(t)=A_{d} e_{x_{d}}(t)-B_{d} k C_{d} e_{x_{d}}\left(t-\tau_{1}\right),
$$


or equivalently,

$$
\left[\begin{array}{c}
\dot{e}_{x_{d}}(t) \\
e_{y}\left(t+\tau_{1}\right)
\end{array}\right]=\left[\begin{array}{cc}
A_{d} & -B_{d} k \\
C_{d} & 0
\end{array}\right]\left[\begin{array}{c}
e_{x_{d}}(t) \\
e_{y}(t)
\end{array}\right]
$$

Consider now a state space realization of system (6). This dynamics can be written in state space form as,

$$
\begin{aligned}
{\left[\begin{array}{c}
\dot{x}(t) \\
y(t-\tau)
\end{array}\right]=} & {\left[\begin{array}{cc}
A & -B k \\
C & 0
\end{array}\right]\left[\begin{array}{l}
x(t) \\
y(t)
\end{array}\right] } \\
& +\left[\begin{array}{c}
B \\
0
\end{array}\right] u(t)
\end{aligned}
$$

with,

$$
\begin{aligned}
& A=\left[\begin{array}{ccccc}
a & 0 & 0 & \cdots & 0 \\
1 & -b_{1} & 0 & \cdots & 0 \\
0 & 1 & -b_{1} & \cdots & 0 \\
\vdots & \vdots & \vdots & \ddots & \vdots \\
0 & 0 & 0 & \cdots & -b_{m}
\end{array}\right], B=\left[\begin{array}{c}
\gamma \\
0 \\
0 \\
\vdots \\
0
\end{array}\right] \\
& C=\left[\begin{array}{lllll}
0 & 0 & 0 & \cdots & 1
\end{array}\right]
\end{aligned}
$$

Comparing (23) and (22) it is clear that Lemma 1 can be applied to system (22). Hence the result of the theorem follows.

\subsection{Proposed Control Scheme}

Using the signals $\hat{\omega}_{1}(s)$ and $\hat{\omega}_{2}(s)$ from the proposed observer-predictor scheme, the ideas depicted in Section 2 can be implemented, i.e., $\hat{\omega}_{2}(s)$ is used in order to decouple the recycling path while $\hat{\omega}_{1}(s)$ can be used to design a controller $J(s)$ that assures the closed-loop stability.

In order to achieve the above purposes, the general control law can be set as,

$$
U(s)=J(s)\left(R(s)-\widehat{\omega}_{1}(s)\right)-\widehat{\omega}_{2}(s) .
$$

This is illustrated in Fig. 6. Notice that the controller $J(s)$ should be designed based on the delay free model $G_{1}(s)$ due to the use of the estimated signal $\omega_{1}(s)$.

Remark 5 Notice that any controller $J(s)$ that stabilizes the delay free model $G_{1}(s)$ with a simple unitary feedback allows stabilizing the closed-loop system shown in Fig. 6 since the convergence of the signals $\omega_{1}(s)$ and $\omega_{2}(s)$ has been assured.

On the other hand, from the design controller $(J(s))$ viewpoint there is not restriction on the magnitude of the delay term $\tau_{1}$, however the estimation of the variable $\omega_{1}(s)$ is possible when $\tau_{1}<\frac{1}{a}-\sum_{i=1}^{m} \frac{1}{b_{i}}$ (Theorem 4 ), therefore this is the only restriction on $\tau_{1}$.

The previous ideas can be summarized in the following result. 


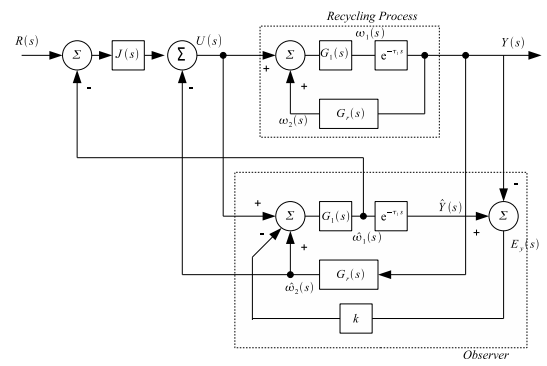

Figure 6: Proposed control scheme for recycling system (2).

Theorem 6 Consider the observer-based control scheme depicted in Figure 6. Then, there exists a controller $J(s), P I / P I D$, such that the closed-loop system is stable if and only if

$$
\tau_{1}<\frac{1}{a}-\sum_{i=1}^{m} \frac{1}{b_{i}}
$$

for $i=1,2 \ldots, m$.

Proof. Consider the observer scheme shown in Figure 6. From Theorem 4, an adequate estimation $\hat{\omega}_{j}(s)$, of the signals $\omega_{j}$ are assured if and only if

$$
\tau_{1}<\frac{1}{a}-\sum_{i=1}^{m} \frac{1}{b_{i}}
$$

for $i=1,2 \ldots, m$. On the other hand, by Lemma 3 , it is possible to find a controller, PI/PID, such that the closed-loop system is stable if and only if $0<\frac{1}{a}-\sum_{i=1}^{m-p} \frac{1}{b_{i}}$ for PI, or $0<\sqrt{\frac{1}{a^{2}}+\sum_{i=1}^{m} \frac{1}{b_{i}^{2}}}+\frac{1}{a}-\sum_{i=1}^{m} \frac{1}{b_{i}}$ for PID controller. Then we can conclude that the closed-loop system of Figure 6 is stable if and only if

$$
\tau_{1}<\frac{1}{a}-\sum_{i=1}^{m} \frac{1}{b_{i}}
$$

Notice that the observer and the control are designed separately then the closedloop system (Figure 6) with the dynamic measurement feedback is stable. Such assertion is valid since the separation principle holds for the proposed linear observer based control. In this case the control and observer systems are stable, satisfying the above mentioned condition.

The following result is concerned with the tracking and disturbance rejection problem for step inputs by considering a PI or PID controller. Here it is assumed that the used PI/PID controller stabilizes the delay free model $G_{1}(s)$ (see Theorem 6). In this way, the general control law (24) is modified as,

$$
U(s)=J(s)\left(R(s)-\widehat{\omega}_{1}(s)+E_{y}(s)\right)-\widehat{\omega}_{2}(s)
$$

where $E_{y}(t)=\hat{y}(t)-y(t)$ and $J(s)$ is a PI/PID controller). 


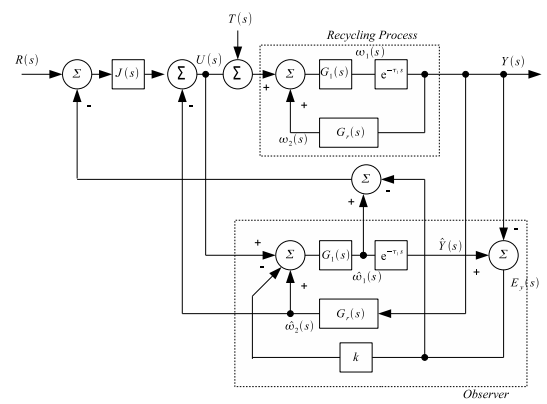

Figure 7: Proposed control scheme with $J(s)$ being PI/PID controller.

Corollary 7 Consider the observer scheme shown in Fig. \%. Then, if the condition of Theorem 6 is satisfied the tracking of step input references and rejecting step disturbances properties are achieved i.e.,

$$
\lim _{t \rightarrow \infty} y(t)=\rho,
$$

where $R(s)=\rho / s$ is the step input reference and $T(s)=0$, and

$$
\lim _{t \rightarrow \infty} y(t)=0,
$$

where $R(s)=0$ and $T(s)$ is a step input disturbance.

Proof. Consider the observer scheme shown in Fig. 7 and the output response expressed as,

$$
Y(s)=G_{i n} R(s)+G_{p e r t}(s) T(s)
$$

where, $G_{i n}$ and $G_{p e r t}$ are transfer functions of the output with respect to the input reference and disturbance input, respectively. First, the tracking step reference property is addressed. Thus, consider

$$
G_{\text {in }}=\frac{G_{d} J\left(1+k G_{1} e^{-s \tau_{1}}\right)}{J\left(G_{d}+G_{1}\left(1-e^{-s \tau_{1}}+k G_{d}\right)\right)+k G_{1} e^{-s \tau_{1}}+1}
$$

The stability of the transfer function (28) can be assured by satisfying the condition of Theorem 6, i.e.,

$$
\tau_{1}<\frac{1}{a}-\sum_{i=1}^{m} \frac{1}{b_{i}}
$$

Therefore the Final Value Theorem with $R(s)=\rho / s$ to the transfer function (28) can be applied, obtaining,

$$
\lim _{t \rightarrow \infty} y(t)=\lim _{s \rightarrow 0} s Y(s)=\lim _{s \rightarrow 0} s \frac{N_{1}(s)}{D_{1}(s)} \frac{\rho}{s}
$$


with,

$$
\begin{gathered}
N_{1}(s)=G_{d} J\left(1+k G_{1} e^{-s \tau_{1}}\right) \\
D_{1}(s)=J\left(G_{d}+G_{1}\left(1-e^{-s \tau_{1}}+k G_{d}\right)\right)+k G_{1} e^{-s \tau_{1}}+1
\end{gathered}
$$

Substituting $G_{d}(s), G_{1}(s)$ given in (2a) and $J(s)$ as a PI or PID controller, the result follows

$$
\lim _{t \rightarrow \infty} y(t)=\lim _{s \rightarrow 0} s Y(s)=\rho
$$

Now the disturbance rejection property is analyzed. Consider

$$
G_{p e r t}=\frac{G_{d}+G_{1} J G_{d}+k G_{1} G_{d} e^{-s \tau_{1}}-G_{1} J G_{d} e^{-s \tau_{1}}}{J\left(G_{d}+G_{1}\left(1-e^{-s \tau_{1}}+k G_{d}\right)\right)+k G_{1} e^{-s \tau_{1}}+1}
$$

The stability of the transfer function (33) can be assured by satisfying the condition of Theorem 6 , i.e.,

$$
\tau_{1}<\frac{1}{a}-\sum_{i=1}^{m} \frac{1}{b_{i}}
$$

Therefore the Final Value Theorem with $T(s)=\eta / s$, to the transfer function (33) can be applied, obtaining,

$$
\lim _{t \rightarrow \infty} y(t)=\lim _{s \rightarrow 0} s Y(s)=\lim _{s \rightarrow 0} s \frac{N_{2}(s)}{D_{2}(s)} \frac{\eta}{s}
$$

with

$$
\begin{gathered}
N_{2}(s)=G_{d}+G_{1} J G_{d}+k G_{1} G_{d} e^{-s \tau_{1}}-G_{1} J G_{d} e^{-s \tau_{1}} \\
D_{2}(s)=J\left(G_{d}+G_{1}\left(1-e^{-s \tau_{1}}+k G_{d}\right)\right)+k G_{1} e^{-s \tau_{1}}+1 .
\end{gathered}
$$

Substituting $G_{d}(s), G_{1}(s)$ given in (2a) and $J(s)$ as a PI or PID controller, we have,

$$
\lim _{t \rightarrow \infty} y(t)=\lim _{s \rightarrow 0} s Y(s)=0
$$

Now, from the previous discussions and results, the proposed methodology can be summarized as follows.

Procedure 8 To obtain the complete control scheme proposed in Fig. 6:

1. Check assumptions of Theorem 4, i.e., $G_{r}(s)$ a stable transfer function and $G_{d}(s)$ of the form (2a)

2. Check that the necessary and sufficient condition in Theorem 4 i.e, $\tau_{1}<$ $\frac{1}{a}-\sum_{i=1}^{m} \frac{1}{b_{i}}$ is satisfied.

3. Choose the parameter $k$. This can be done by considering (10) and (11). 
4. Design the controller $J(s)$ based on the delay free model of the direct path, $G_{1}(s)$. A PI/PID control based strategy can be considered (see Theorem 6 and Corollary 7). Notice that as the internal states are estimated, it is also possible to use an estimated state feedback instead of the controller $J(s)$.

\subsection{Application of the method to the direct path with possible $L H P$ zeros.}

This subsection presents how to apply the methodology developed in previous section in order to deal with recycling systems when the direct path includes $L H P$ (stable) zeros. In particular the stabilization problem is solved for this class of systems. Therefore, $G_{d}(s)$ and $G_{r}(s)$ are now defined as,

$$
\begin{aligned}
G_{d}(s) & =\frac{\alpha\left(s+z_{1}\right) \ldots\left(s+z_{p}\right)}{(s-a)\left(s+b_{1}\right) \ldots\left(s+b_{m}\right)} e^{-\tau_{1} s}, \\
G_{r}(s) & =G_{2}(s) e^{-\tau_{2} s}=\frac{N(s)}{D(s)} e^{-\tau_{2} s},
\end{aligned}
$$

with $p \leq m$ and $z_{1}, \ldots, z_{p}>0$, i.e., left half plane $(L H P)$ zeros in the direct path of the recycling system. Before providing the observation strategy designed for system (37) some further ideas are depicted. Consider the partition of $G_{d}(s)$ as,

$$
G_{d}(s)=G_{1 a}(s) e^{-\tau_{1} s} G_{1 b}(s)
$$

where,

$$
\begin{aligned}
G_{1 a}(s) & =\frac{\alpha}{(s-a)\left(s+b_{1}\right) \ldots\left(s+b_{m-p}\right)}, \\
G_{1 b}(s) & =\frac{\left(s+z_{1}\right) \ldots\left(s+z_{p}\right)}{\left(s+b_{m-p+1}\right) \ldots\left(s+b_{m}\right)},
\end{aligned}
$$

Now, Fig. 8 shows how to extend the ideas presented in Section 2 to the recycling system given by (37). From Fig. 8, the estimation of $\omega_{2}(s)$ as well as $\omega_{1}(s)$ are required in order to stabilize the process. However, from our assumptions, the measure of these variables are not available from the process and an observer to estimate the required signals is used. The proposed observer scheme is shown in Fig. 9.

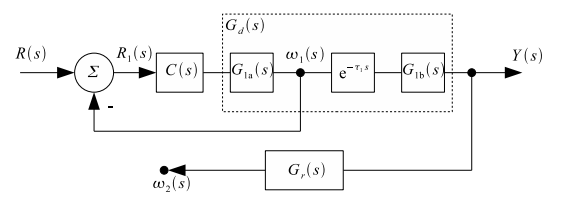

Figure 8: Main idea of stabilization. 


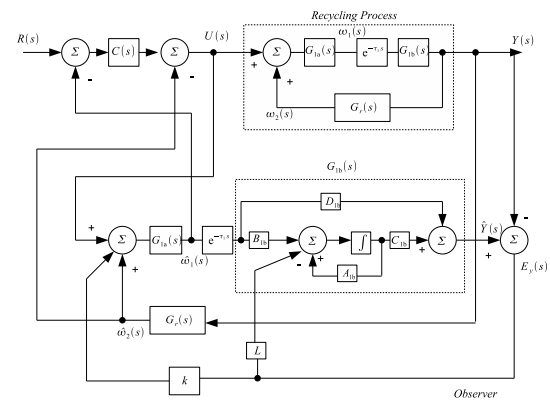

Figure 9: Proposed control/observer strategy for recycling system (37).

Theorem 9 Consider the observer-predictor scheme shown in Fig. 9, assuming $G_{r}(s)$ is a stable transfer function and $G_{d}(s)$ defined as in (37a). There exists a constant $k$ and a vector $L$ such that

$$
\lim _{t \rightarrow \infty}\left[\omega_{j}-\widehat{\omega}_{j}\right]=0,
$$

for $j=1,2$. , if and only if

$$
\tau_{1}<\frac{1}{a}-\sum_{i=1}^{m-p} \frac{1}{b_{i}}
$$

for $i=1,2 \ldots, m$.

Sketch of the proof. The proof of this Theorem is similar to the proof of Theorem 4 by using Lemma 2 instead of Lemma 1. Thus, the error dynamics of the scheme shown in Fig. 9 are obtained, which are compared with the dynamics analyzed in Lemma 2. As both dynamics are the same, we conclude that the error convergence conditions are given by the stability conditions of the closed-loop system presented in Lemma 2.

From Theorem 9, we have adequately estimated the internal signals $\widehat{\omega}_{1}(s)$ and $\widehat{\omega}_{2}(s)$. In order to stabilize the recycling system given by (37), the ideas provided previously in Fig. 8 are applied by using the following control law,

$$
U(s)=C(s)\left(R(s)-\widehat{\omega}_{1}(s)\right)-\widehat{\omega}_{2}(s)
$$

Note that in (41), the controller $C(s)$ should be designed for the delay free plant $G_{1 a}(s)$ as shown Fig. 8 (for instance, a proportional controller). However, notice that in this case it is not possible to implement $C(s)$ as a PI or PID controller in order to achieve tracking step references, since in the direct path of the recycling plant we have an additional dynamic due to $G_{1 b}(s)$. In this way, the control-observer strategy is shown in Fig. 9 and achieves the stabilization of the recycling system when the direct path consider LHP zeros. Now, the application of the method for this class of systems can be summarized in the following result. 
Procedure 10 To get the whole control scheme proposed in Fig. 9, is,

1. Check assumptions of Theorem 4 are satisfied, i.e., $G_{r}(s)$ a stable transfer function and $G_{d}(s)$ of the form (37a).

2. Check that the necessary and sufficient condition in Theorem 4, i.e., $\tau_{1}<$ $\frac{1}{a}-\sum_{i=1}^{m-p} \frac{1}{b_{i}}$ is satisfied.

3. Design of the observer parameter $L$, by considering a traditional output injection to $G_{1 b}(s)$. The parameter $L$ should be computed such that the relocated poles of $G_{1 b}(s)$ are set at $\left\{-z_{1},-z_{2} \ldots-z_{p}\right\}$. This can be done by using the commands acker or place from the package MATLAB.

4. Choose observer parameter $k$ from (11). This can be done by taking into account only $G_{1 a}(s) e^{-\tau_{1} s}\left(G_{1 a}(s)\right.$ given by (39a)).

5. Design the controller $C(s)$ based on the delay free model of the direct path, $G_{1 a}(s)$, for instance a proportional controller.

\section{SIMULATION RESULTS}

In this section, two academic examples show the performance of observer based control strategy previously proposed. In examples the procedure given have to be followed namely:

1. Check assumptions of Theorem $4 / 9$ are satisfied.

2. Check that the necessary and sufficient condition in Theorem 4/9.

3. Design of the observer parameter(s).

4. Design of the controller $J(s) / C(s)$ based on the delay free model of the direct path, $G_{1}(s) / G_{1 a}(s)$.

\section{$5.1 \quad$ Example 1.}

Consider the delayed recycling system of the form (1) with,

$$
G_{d}(s)=\frac{1}{(s-1)(s+10)} e^{-0.5 s}, \quad G_{r}(s)=\frac{1}{s+1} e^{-2 s} .
$$

Following the procedure described by Procedure 8 , it is verified that $G_{r}(s)$ is a stable transfer function and $G_{d}(s)$ is of the form of (2a). Also we see that the condition $0.5=\tau_{1}<\frac{1}{a}-\sum_{i=1}^{m} \frac{1}{b_{i}}=0.9$ is satisfied. Then, the single observer parameter $k=12$ is chosen from (11). Finally, the controller $J(s)$ is proposed as 
a two degree of freedom PID [24], which is designed based on delay free model $G_{1}(s)$. Such controller is implemented as,

$$
U(s)=R(s) G_{f f}(s)-G_{c}(s) \widehat{\omega}_{1}(s)-\widehat{\omega}_{2}(s)
$$

with

$$
\begin{gathered}
G_{f f}(s)=160\left(0.3+\frac{1.6}{s}+0.1 s\right), \\
G_{c}(s)=160\left(1+\frac{1.6}{s}+0.1 s\right) .
\end{gathered}
$$

Now, in order to evaluate the output signal evolution some numerical simulations are presented. It is considered a positive unit step input and initial conditions in $G_{1}(s)$ of the process and the observer of magnitude 0.4 units and 0.2 units, respectively. Also an unit step disturbance $T(s)$ acting at 15 sec. is considered. In Fig. 10, a solid line shows the output response when it is considered the exact knowledge of the model parameters; a dashed line presents the output signal when the time delays $\tau_{1}$ and $\tau_{2}$, are increased by $8 \%$ and $15 \%$, respectively. Then, without disturbance $T(s)$ Fig. 11 shows the corresponding output estimation error $e_{y}(t)$. From Fig. 10 and Fig. 11 it can be seen the observer predictor convergence and the nice behavior of the control based on estimated signals.

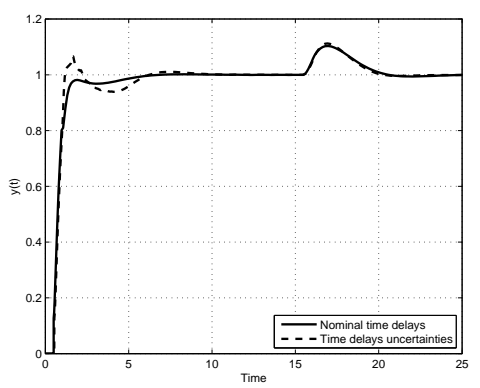

Figure 10: Output signal with different initial condition in process and observer.

\subsection{Example 2.}

Consider the recycling system (1) where,

$$
\begin{aligned}
G_{d}(s) & =\frac{(s+3)(s+5)}{(s-1)(s+8)(s+1)(s+2)} e^{-0.6 s} \\
G_{r}(s) & =\frac{1}{s+1} e^{-s} .
\end{aligned}
$$

As $L H P$ zeros in the direct path of recycling system are considered, the method developed in Subsection 4.3 is used. Following the procedure of Procedure 10, 


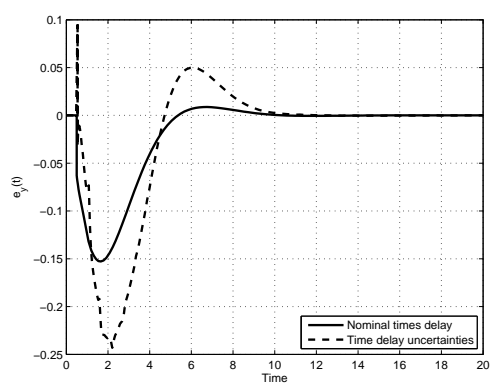

Figure 11: Estimation error $e_{y}(t)$ by considering different initial condition in process and observer.

we see that the assumptions given by Theorem 4 are satisfied, i.e. $G_{r}(s)$ is a stable transfer function and $G_{d}(s)$ is of the form of (37a). Thus we define,

$$
\begin{aligned}
G_{1 a}(s) & =\frac{1}{(s-1)(s+8)} \\
G_{1 b}(s) & =\frac{(s+3)(s+5)}{(s+1)(s+2)} .
\end{aligned}
$$

Then, the established condition in Theorem 4 is also satisfied since $0.6=\tau_{1}<$ $\frac{1}{a}-\sum_{i=1}^{m-p} \frac{1}{b_{i}}=0.875$.

Now, in order to design the observer parameter $L$, an output injection to $G_{1 b}(s)$ is applied. In this way, consider a possible state space representation of $G_{1 b}(s)$ as,

$A_{1 b}=\left[\begin{array}{cc}-3 & -1.4142 \\ 1.4142 & 0\end{array}\right], B_{1 b}=\left[\begin{array}{l}1 \\ 0\end{array}\right], C_{1 b}=\left[\begin{array}{ll}5 & 9.1924\end{array}\right]$ and $D_{1 b}=1$. The vector $L$ should be computed such that the poles of $G_{1 b}(s)$ are relocated at $\{-3,-5\}$, i.e.,

$$
\sigma\left(s I-A_{1 b}-L C_{1 b}\right)=\{-3-5\}
$$

where $\sigma($.$) represents the eigenvalues of. As a result we obtain L=\left[\begin{array}{l}1 \\ 0\end{array}\right]$. The second observer parameter $k$ can be obtained from (11) with $G_{1 a}(s) e^{-\tau_{1} s}$, in such case we have $8<k<12.8$, to simulation $k=10$ is used. Finally, as mentioned before a proportional controller $C(s)$ can be designed with a Nyquist or Root Locus diagrams based on delay free model $G_{1 a}(s)$. In this way, we consider $C(s)=K_{1}$ as a static controller. Therefore, it is obtained $K_{1}>8$ in order to stabilize, and $K_{1}=20$ is chosen to the simulation experiments.

In order to evaluate the output signal performance, an initial condition in the states of $G_{1 a}(s) G_{1 b}(s)$ of magnitude 0.4 units is regarded. In Fig. 12 a solid line shows the output response when it is considered the exact knowledge of the model parameters; a dashed line presents the output signal when the process 
time delays $\tau_{1}$ and $\tau_{2}$, are increased by $5 \%$. Also in Fig. 13 the estimation error at the output $e_{y}(t)$ is presented.

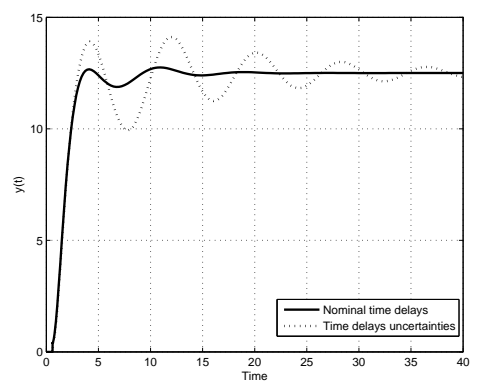

Figure 12: Stabilization of recycling system, Example 2.

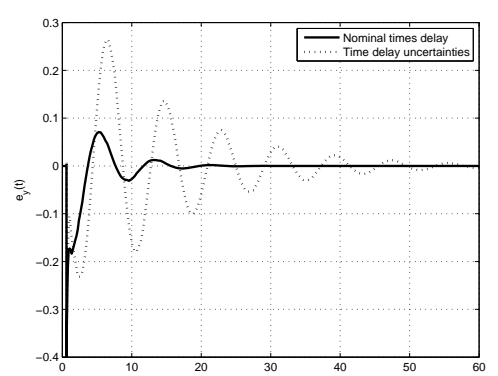

Figure 13: Output error signal, Example 2.

\section{CONCLUSIONS}

Using recycle in unstable processes with significant time delay leads to a challenging control problem. In this work this problem has been addressed for the case of one unstable pole, $m$ stable poles, and possibly $p L H P$ zeros with significant delay in the direct path, which is a more general case than the one analyzed in [16] and [17]. Results with respect to step tracking reference and step disturbance rejection are also presented. Explicit conditions for the construction of a stabilizing observer based controller scheme for such class of systems are presented. The observer-prediction strategy is used to estimate some internal variables of the process that are used to: i) remove the dynamics of recycling path in the process and ii) design a stabilizing control law for the free delay model of the direct path. Simulation results have been presented in order to show the performance of the method under different circumstances. 


\section{References}

[1] Luyben W.L., Tyreus B. D. and M. L. Luyben, Plantwide control, McGraw Hill, New York (1999).

[2] Luyben M. L. and W. L. Luyben, Essentials of process control, McGraw-Hill, (1997).

[3] Luyben W. L., Temperature control of autorefrigetater reactor, J. Process Control, vol. 9, (1999) pp 301.

[4] Taiwo O., The design of robust control systems for plants with recycle, Int. J. Control, vol. 43(2) (1986), pp 671.

[5] Scali C. and F. Ferrari, Performance of control systems based on recycle compensators in integrated plants, J. Process Control, vol. 9, (1999), pp 425.

[6] Lakshminarayanan S. and H. Takada, Empirical modelling of processes with recycle: some insights via case studies, Chem. Eng. Sci., vol. 56, (2001), pp 3327.

[7] Kwok K.E., Chong-Ping. M. and G. A. Dumont, Seasonal model based control of processes with recycle dynamics, Ind. Eng. Chem. Res., vol. 40, (2001), pp 1633.

[8] Nesimioglu B.S. and M.T. Soylemez, A simple derivation of all stabilizing proportional controllers for first order time-delay systems, Asian Journal Of Control, vol. 14, (2010), pp 598-604.

[9] Papadourakis A., Doherty M. F. and J.M. Douglas, Approximate dynamic models for chemical process systems, Ind. Eng. Chem. Res., vol. 28, (1989), pp 546-552.

[10] Malek-Zavarei M. and M. Jamshidi, Time-Delay systems. Analysis, optimization and applications, NorthHolland, The Netherlands, (1987).

[11] Hugo A.J., Taylor P.A. and J.D. Wright, Approximate dynamic models for recycle systems, Ind. Eng. Chem. Res., vol. 35, (1996), pp 485-487.

[12] Del Muro-Cuellar B., Velasco-Villa M., Puebla H. and J. Alvarez-Ramirez, Model approximation for Dead-Time Recycling Systems, Ind. Eng. Chem. Res., vol. 44, (2005), pp 4336-4343.

[13] Samyudia Y.K., Kadiman K., Lee O.L. and I.T. Cameron, Gap metric based control of processes with recycle systems, Proccedings of ADChEM, Pisa-Italy, (2000), pp 497-502.

[14] Astrom K.J., Hang C.C. and B.C. Lim, A new Smith predictor for controlling a process with an integrator and long dead time, IEEE Trans. Aut. Control, vol. 39(2), (1994), pp 343-345. 
[15] Maza-Casas L., Velasco-Villa M. and J.A. Alvarez-Gallegos, On the state prediction of linear systems with time-delays in the input and the state, Proc. 38th. IEEE Conf. On Decision and Control, USA, December, (1999), pp. 239-244.

[16] Marquez-Rubio J.F., del Muro-Cuéllar B., Velasco-Villa M. and D. CortésRodríguez, Observer scheme for linear recycling systems with time delays, American Control Conference, San Francisco, USA, (2011).

[17] Marquez-Rubio J.F., del Muro-Cuéllar B., Velasco-Villa M., CortésRodríguez D. and O. Sename, Control of delayed recycling systems with unstable first order forward loop, J. of Process Control, vol. 22, (2012), pp 729-737

[18] Marquez-Rubio J.F., del Muro-Cuéllar B. and O. Sename, Control of delayed recycling systems with an unstable pole at forward loop, American Control Conference, Montréal, Canada, (2012).

[19] Del Muro-Cuellar B., Marquez-Rubio J.F., Velasco-Villa M., AlvarezRamirez J. and H. Puebla, Stabilization and control of unstable first order linear delay systems, Internat. Conf. on Modelling, Shangai, (2008).

[20] Pedraza-Beltrán Y., González-Najera O. and B. del Muro-Cuéllar, Stabilization of High Order Systems with Delay Using a Predictor Schema, 52nd. Midwest MWSCAS IEEE, Cancun Mex. August (2009).

[21] Lee S.C., Wang Q.-G. and Xiang C., Stabilization of all-pole unstable delay processes by simple controllers, Journal of Process Control, Vol. 20, (2010), pp 235-239.

[22] Silva G.J., Datta A. and Bhattacharyya S.P. PID controllers for time-delay systems, Birkhuser, Boston (2005).

[23] Verriest E., Sename O. and P. Pepe, Robust observer controller for delaydifferential system, Proc. of the IEEE International Conference on Desicion and Control, Las Vegas, USA, December, (2002).

[24] Astrom K. J. and T. Hagglund, PID Controllers, theory, design and tuning, International Society for Measurement and Control, New York, (1995). 